\title{
SOBRE LOS SUPUESTOS LESBISMOS DEL DIALECTO CIRENAICO*
}

\begin{abstract}
It has been supposed the existence of common morphological and phonetic features between Cyrenaean and Lesbian: the phonetic development of secondary -ns-; the dative plural of consonantal stems in - $\varepsilon \sigma \sigma l$; the infinitive type $\delta$ เ $\delta \omega v$; the perfect par-

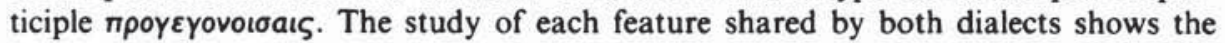
great difficulties that all of them imply and induces to question the existence of Lesbian features in this dialect at any time: the treatment of the secondary cluster $-n s$ to -is- does not occur in final position, like in Lesbian and cannot only be explained from Lesbian; the agreement of the $-\varepsilon \sigma \sigma$ เ datives in ${ }^{*}-\tilde{e} u$ does not really exist and the - $\varepsilon \sigma \sigma$ t forms do not occur in Cyrenaean regularly in other stems, as opposed to Lesbian; the infinitive $\delta\llcorner\delta \omega v$ can be explained as a transfer of this form to the inflection

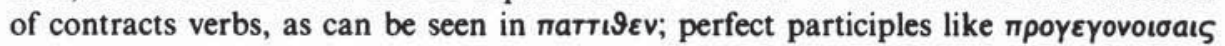
occur sporadically in other dialects also and could even be an artificial form.
\end{abstract}

\section{Coincidencias del dialecto cirenaico con el lesbio}

Como es sabido ', Cirene es una colonia de la isla de Tera, fundada en el año 630 a. C., cuya lengua presenta, al menos en apariencia ${ }^{2}$, notables divergencias con respecto a su metrópoli ${ }^{3}$. Entre éstas, figuran las

* Los siglos o años a los que pertenecen las inscripciones son anteriores a nuestra era, salvo mención expresa.

I F. Chamoux, Cyrène sous la monarchie des Battiades, Paris 1953.

2 La falta de documentos de los siglos vi/v e incluso iv a. C. en Tera dificulta la interpretación de las grafias en este dialecto: .... JWoa IG XII, 3S. n. ${ }^{\circ} 1289$ (s. Iv),

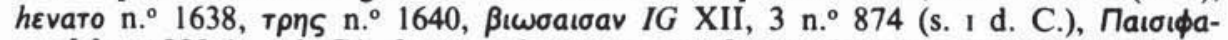

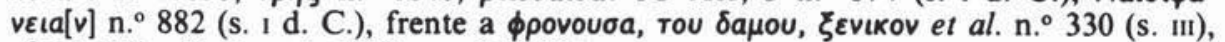
cf. M. Bile, Verbum 2, 1979, pp. 161-162.

${ }^{3}$ Asi, las vocales resultantes de los alargamientos compensatorios y de las con-

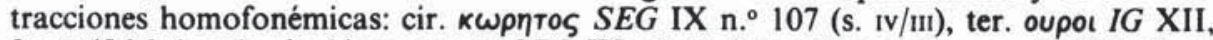
$3 \mathrm{n}^{\circ} 436.1$ (s. Iv), cir. Nikootpatw SEG IX n. ${ }^{\circ} 11.2$ (s. Iv), ter. Гpıvvou IG XII, $3 \mathrm{n} .{ }^{\circ}$ 330.3 (s. III). El cirenaico presenta también características exclusivas con respecto a los restantes dialectos griegos, favorecidas sin duda por su aislamiento geográfico, como el nom. ac. pl. de los temas en -eu-: o tapes $S E G$ IX n. 5 (s. II/I), tos tapes SEG IX n. 4.31 (16/5), Apoivoes SEG XXVI n. 1817.73 (s. ı). 
coincidencias entre el cirenaico y los dialectos eolios interpretadas habitualmente como debidas a un sustrato eolio o bien como desarrollos paralelos $^{4}$. Recientemente ${ }^{5}$, reavivando asi el problema, se ha subrayado que la presencia de un elemento específicamente lesbio ( $y$, por tanto, no de un sustrato eolio) en Cirene puede apoyarse en una serie de coincidencias de entre las que ninguna es definitiva por si sola pero que, tomadas en conjunto, pueden ser significativas: (a) la evolución del grupo -ns- secundario; (b) los dativos en - $\varepsilon \sigma \sigma \iota ;$ (c) el infinitivo $\delta \iota \delta \omega v$; (d) el

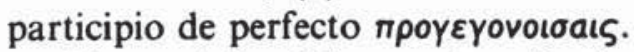

El propósito del presente artículo es analizar cada uno de estos rasgos y plantear si realmente apoyan la existencia de un componente lesbio en el dialecto de Cirene.

\section{La evolución del grupo -ns- secundario}

El resultado del grupo -ns- secundario (tipos * pansa, part. *-onsa <*-ontya) y -ns originario (ac. pl. -ons, -ans) presenta en lesbio, frente al resto de los dialectos griegos, un tratamiento especial: -Ens->-Eis-; $-E n s>-E i s$, con el que coinciden parcialmente el eleo (sólo en posición final -Eis) y el cirenaico (sólo en interior -Eis-). El resultado en lesbio se ha explicado (a) como debido a la existencia de una nasal palatalizada o (b) como debido en última instancia a la nasalización de la vocal en contacto con la nasal (no palatalizada). El aumento de la energía articulatoria de la sonante explicaría el proceso (a) en lesbio ${ }^{6}$, es decir, provocaría la palatalización de la nasal y su posterior despalatalización: "pantia $>$ *panta $>{ }^{*}$ pant' $a>{ }^{*}$ pansa $>$ pansa $>$ paisa $(<\pi a i \sigma a>)$. Al margen de que la nasal resultante de este proceso represente un alófono o un auténtico fonema, la palatalización no estaría necesariamente condicionada por el contexto y por ello podría producirse tanto en *pansa como en ${ }^{*}$ tons $^{7}$.

4 A favor de un sustrato eolio, A. Braun, RFIC 60, 1932, pp. 181-193 y 309 331; C. D. Buck, Cl. Ph. 41, 1946, pp. 129-134; como desarrollos paralelos, G. Devoto, RFIC 56, 1928, pp. 365-403.

S. L. Garcia Ramón, Minos 16, 1977, pp. 203-204.

- M. S. Ruipérez, Actas del III Congreso Español de Estudios Clásicos, III, Madrid 1968, pp. 193-199, para quien la nasal palatal es un fonema en lesbio, p. 197; Cl. Brixhe, Etrennes de septantaine. Travaux ... offerts à M. Lejeune, París 1978, pp. 65-73, y $B S L 74,1,1979$, pp. 241-251.

Una opinión que no comparto es la que afirma que este fenoméno estaria restringido originariamente al grupo -ns- en donde la palatalización de la nasal se debería a su contacto con la africada resultante de la evolución del grupo -ti-: "pan-

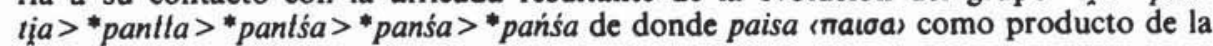


La explicación alternativa (b) postula la realización de vocoides nasalizados ${ }^{8}$ en todos los dialectos: * pant $a>{ }^{*}$ pansa $>{ }^{*}$ paz̃sa $>$ paisa (por desnasalización de *paz̃sa). En todos ellos, salvo en lesbio y en chipriota en el que ki-jo-na-u, Kadmos 19, 1980, p. 65, s.2.4. (Pafos, ca. 325309 a. C.) e $I C S 90,4$, parece sugerir una evolución - $s^{->-}-u s_{-}$, la pérdida de la resonancia nasal produciría el alargamiento de la vocal correspondiente. La hipótesis (b) puede ser ciertamente válida en casos como el chipriota, el eleo o cualquier otro dialecto, incluido el cirenaico. En todo caso, la verosimilitud de la existencia de fonemas palatales del tipo $/ \mathrm{n}^{\prime} /$ en lesbio propuesta por M. S. Ruipérez parece excluir, al menos para el lesbio, la explicación (b). El problema que queda abierto es, en todo caso, si para el cirenaico es válida la hipótesis (a) o la (b).

La evolución -Ens-<-Eis- se atestigua en Cirene desde el s. IV a. C.:

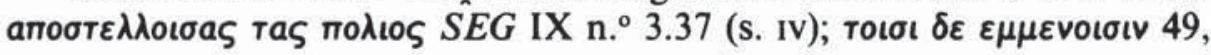

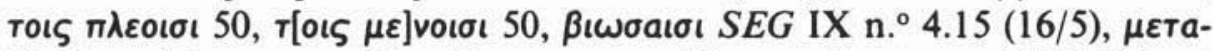

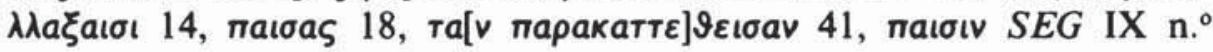

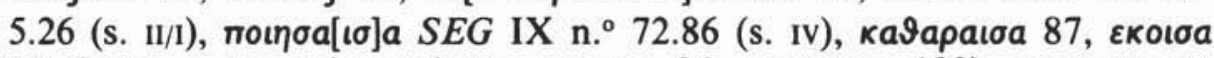

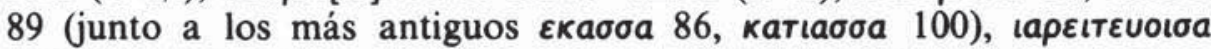
SEG IX n. ${ }^{\circ} 115.5$ (s. III p. C.), umapxorбav $S E G$ XXVI n. ${ }^{\circ} 1817.59$ (s. I),

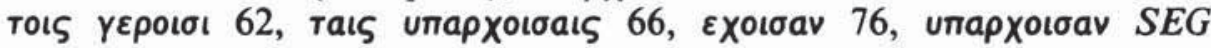

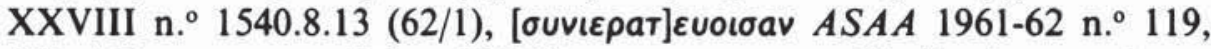

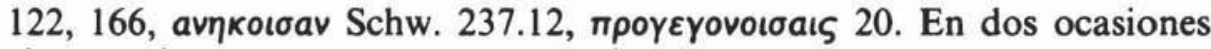
el tratamiento es aparentemente anomalo: por una parte, тоı катоıк-

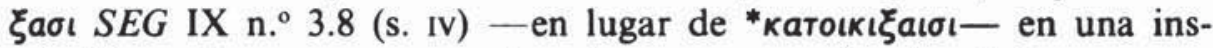
cripción en la que aparecen otras formas que se desvían de la norma y que cabe entender asimismo como extrañas al dialecto ${ }^{9}$ : ol (art.) 5.9, ka-

despalatalización final. En lesbio, *tońs, *tańśs serían creaciones analógicas sobre *pańśa, asi J. Safarewicz, PF 18, 1939, p. 2 (=Linguistic Studies, Parí 1974, p. 107), o bien la oposición -ńs-/-ns- habria desaparecido en favor de -ńś-, así W. Blümel, Die aiolischen Dialekte, Gotinga 1982, p. 105 (coincidiendo únicamente en este punto con Ruipérez, op. cit., p. 197); sin embargo, el resultado de un proceso de despalatalización no puede dar, a su vez, otra palatal, cf. Brixhe, op. cit. 1978, p. 68, y 1979, pp. 243-244, frente a la opinión de E. Risch, Colloquium Mycenaeum, 1979, pp. 267-281 (=Kleine Schriften, Berlin-Nueva York 1981, pp. 549-559).

8 E. Risch, $M H 6$, 1949, p. 22 (=Kleine Schriften, pp. 196-205); A. López Eire, emerita 45, 1977, pp. 313-324; recientemente, el propio Risch, Athlon ... Adrados, I, Madrid 1984, pp. 427-432. Ambos aducen en favor de la evolución -ons $>-\tilde{s}>>$-ous el testimonio de la ley Colonial de Caleo IG IX n. ${ }^{\circ} 718(500 / 475)$ perteneciente a la Lócride Occidental, pero sus argumentos han sido rebatidos convincentemente por J. Méndez Dosuna, Los dialectos dorios del Noroeste: gramática y estudio dialectal, Salamanca 1985 , p. 74.

9 Se trata del juramento de los fundadores; los estudiosos no se ponen de acuerdo en atribuirlo al cirenaico o al dialecto de Tera, cf. M. Bile, op. cit., pp. 154-155. A pesar de que muchas formas son ajenas al cirenaico, otras como anoorendoiras.

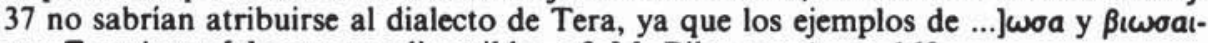
oav חaıљıаveıa[v] son muy discutibles, cf. M. Bile, op. cit., p. 162. 


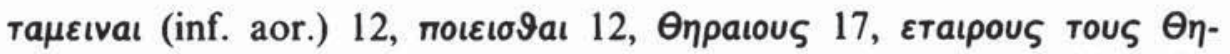

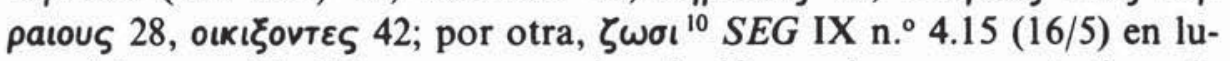
gar del esperable * $\zeta \omega \iota \sigma \iota$, en una inscripción en la que se atestigua la

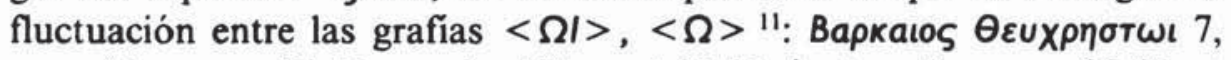

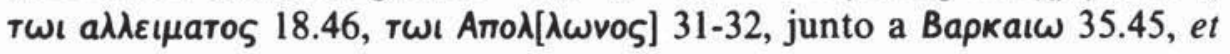
sim.

Las grafias del tipo <naira> no presuponen necesariamente una misma realidad fonética (ni una misma evolución a partir de *-ansa) en los dialectos de Cirene y de Lesbos. Como hemos señalado supra, en este último, la evolución Ens $>$ Eis no es un fenómeno aislado, sino que se encuentra enmarcado dentro de un proceso de palatalización inseparable de la existencia de fonemas líquidos y nasales palatales $\left(/ \mathbf{l}^{\prime} /, / \mathbf{r}^{\prime} /\right.$, $\left./ \mathrm{m}^{\prime} /, / \mathrm{n}^{\prime} /\right)^{12}$. En cambio, en cirenaico no hay indicio alguno de la existencia de tal proceso de palatalización, por lo que el recurso a un elemento lesbio para explicar estas formas estaría plenamente justificado: se trataría de la implantación de un rasgo ajeno al sistema fonológico del cirenaico procedente de otro dialecto. Ahora bien, admitida la hipótesis de un elemento foráneo, habría sido esperable que la influencia se llevara a cabo de forma consecuente y, por tanto, que apareciera en los mismos contextos, es decir, no sólo en -Ens- sino también en -Ens. El hecho de que sólo esté afectado $-n s-$ y no $-n s$ resulta, pues, desconcertante desde este punto de vista y parece descartar la existencia de un elemento especificamente lesbio. Es, en mi opinión, más verosimil que el resto de los dialectos griegos (que parecen no haber conocido un sistema de fonemas sonantes palatalizados) hayan conocido un estadio de nasalización en la evolución de estos grupos; el cirenaico, que debió de encontrarse en esta situación, no se habría separado de esa tendencia común, pero su aislamiento geográfico, al igual que en el caso del chipriota, favoreció su tratamiento parcialmente divergente ${ }^{13}$.

\footnotetext{
10 Devoto, op. cit., p. 381, se basa precisamente en este ejemplo para demostrar un tratamiento distinto del grupo -ns- en este contexto en cirenaico frente al lesbio en donde la evolución es siempre la misma: үрафwı เ IG XII 2 n. ${ }^{\circ} 1.3$.

"Para C. Gallavotti, Maia 15, 1963, p. 455, n. 2, estos genitivos transcritos con $\langle\Omega|>$ serian "come genitivi in -0 e $\mathrm{e}$ in -oı, coesistenti anche in Tessaglia nell'età storica".

${ }_{12}$ Algo similar parece haber ocurrido en tesalio, cf. J. L. García Ramón, CFC 14,1978 , pp. 403-423.

13 De la misma manera deben explicarse los ejemplos del eleo, cf. M. J. García Blanco, Gramática de las inscripciones eleas (Fonética y Morfologia). Tesis inédita, Univ. Santiago de Compostela 1980, II, p. 305, aplicando, sin embargo, la misma hipótesis para el lesbio.
} 


\section{Los dativos en - $\varepsilon \sigma \sigma \mathrm{\iota}$}

La desinencia de dat. pl. atemático - $\varepsilon \sigma \sigma \iota$ en cirenaico está atestiguada en dos inscripciones: $S E G \mathrm{IX} \mathrm{n} .^{\circ} 2$ y $S E G \mathrm{IX} \mathrm{n} .{ }^{\circ} 76$. En la primera,

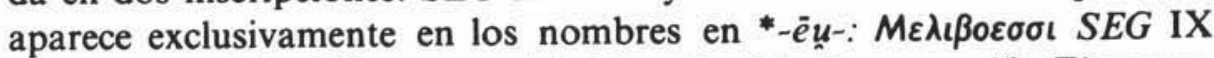

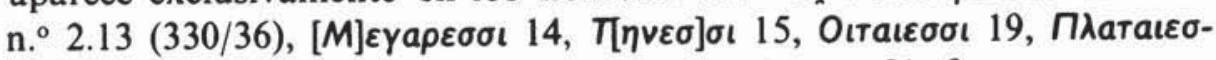

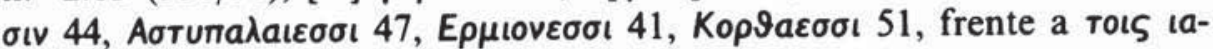

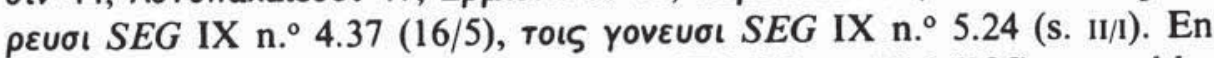

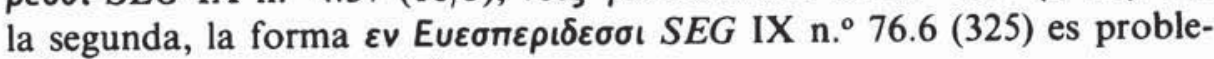
mática, como veremos infra.

Conviene precisar en todo caso que los datos relativos a los restantes temas en cirenaico presentan regularmente la desinencia - $\sigma$ ( no - $\varepsilon \sigma-$

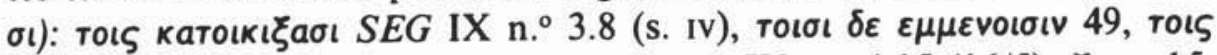

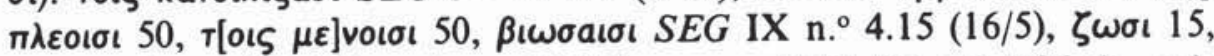

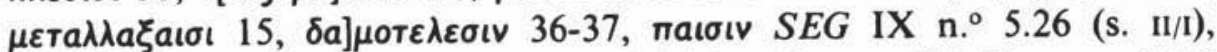

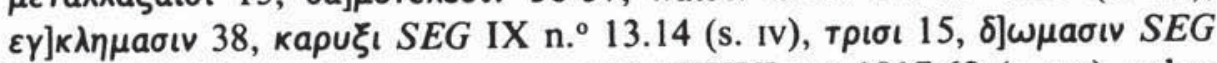

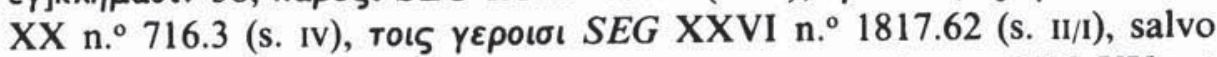

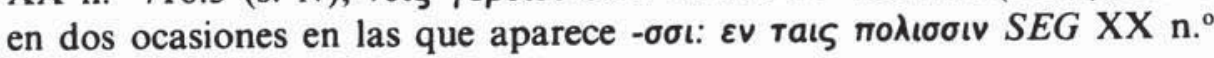

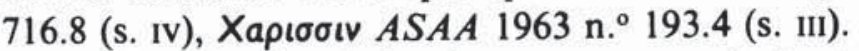

La distribución de las formas en - $\varepsilon \sigma \sigma \iota$ de los temas en -èu- en cirenaico difiere con respecto a la que presenta el lesbio ${ }^{14}$ : (a) en este dialecto - $\varepsilon \sigma \sigma \iota$ es una desinencia ${ }^{15}$ perfectamente distinguible del tema: [ $\beta a$ -

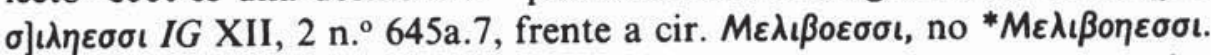
(b) en cirenaico - a diferencia del lesbio- aparece - $\sigma \iota$ (y no - $\varepsilon \sigma \sigma \iota)$ incluso en los nombres en los que la desinencia $-\sigma \iota$ ha producido una desfiguración del tema: así, en los participios $\varepsilon \mu \mu \varepsilon v o เ \sigma \iota v, \pi \lambda \varepsilon o เ \sigma \iota, \mu \varepsilon] v o เ \sigma \iota$ (cf. supra, 2).

La terminación - $\varepsilon \sigma \sigma \iota$ sólo puede ser en principio considerada como

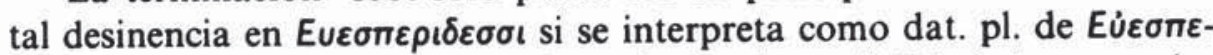
pís (topónimo en -id-). Cabe, sin embargo, la posibilidad de que se in-

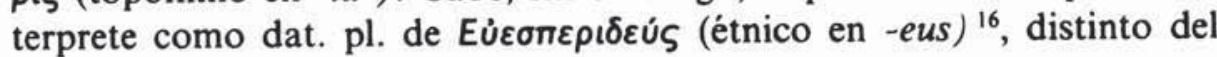

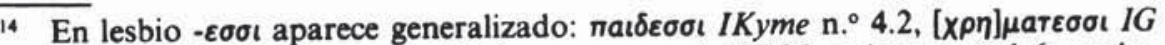

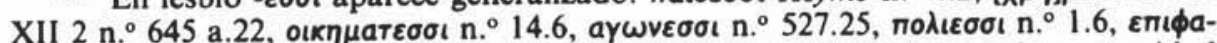

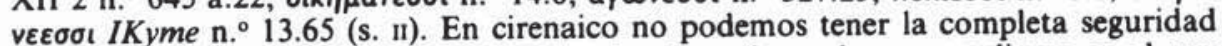
de que - $\varepsilon \sigma \sigma$ เ fuese realmente una desidencia: si partimos de un paradigma en el que

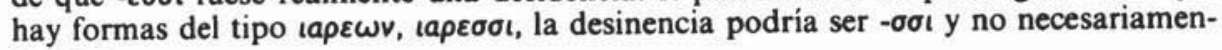
te $-\varepsilon \sigma \sigma$.

is Para un estado completo de la cuestión sobre el origen de los dativos en - $\varepsilon \sigma \sigma$, cf. L. M. Macía, ICEUM, 1984, pp. 305-308.

16 Asi, C. J. Ruijgh, Mnemosyne 11, 1958, p. 101, y P. Wathelet, Les traits éo-

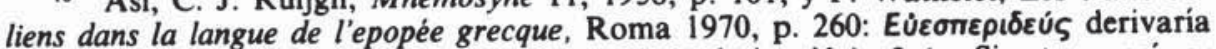

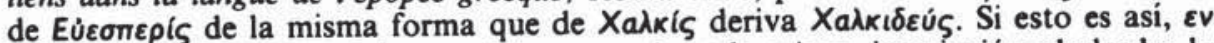

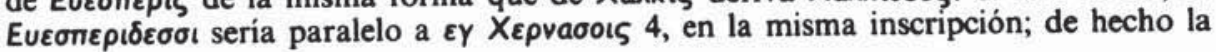




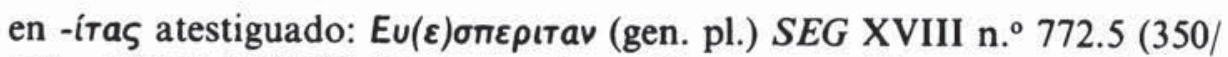
$20)$ y $S G D I 4870$ (1). Dado que los sufijos - $\varepsilon \cup \varsigma$ e -(l)ras para la formación de étnicos son los más productivos en esta época, cabe suponer que pueden haber coexistido en cirenaico ${ }^{17}$.

Parece, pues, que la existencia en Cirene de un dativo en - $\varepsilon \sigma \sigma \iota$ es, cuando menos, dudosa y desde luego su distribución no coincide con la del lesbio. Así pues, al margen del origen de la forma - $\varepsilon \sigma \sigma \iota$ en cirenaico, bien a partir del nom. pl. o ac. pl. de los temas en $-\bar{e} u-{ }^{18}$, bien a partir del dat. pl. de los temas en $-s^{-19}$, dicha forma debe entenderse como independiente del lesbio.

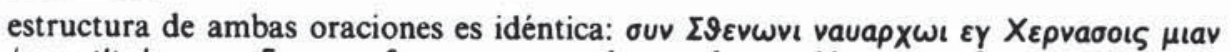

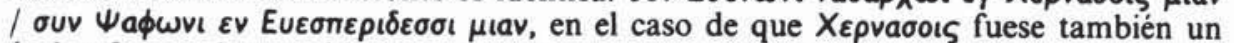

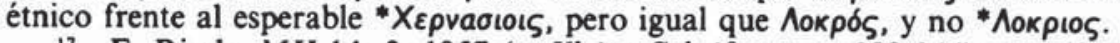

17 E. Risch, $M H$ 14, 2, 1957 (= Kleine Schriften, pp. 133-144), pp. 63-74. Aun rechazando esta argumentación, cabe señalar que la redacción de toda la inscripción parece cuidada, especialmente por el paralelismo de sus oraciones y el sintagma nот'

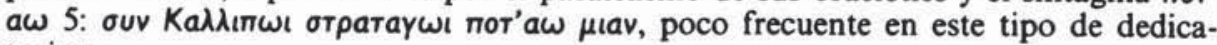
torias.

$18 \mathrm{Si}$ nos basamos en la ecuación analógica -ol/-oı que el nom. ac. pl. de los temas en -eu- atestiguado es $-\varepsilon \varsigma$. Sin embargo, el origen de este nom. ac. es enigmático y ha sido diversamente explicado: - por haplologia doria, R. Günther, IF 32, 1913, p. 379, n. 1 (con un exhaustivo estado de la cuestión). Esta haplología se habria producido también en $\lambda \varepsilon \times 0 \zeta(=\lambda \varepsilon \times \omega \varsigma), S E G$ IX $\mathrm{n}$. 72.109 , frente a $\zeta 00 v$ ( $\zeta \omega \circ v) 69$ y en esto coincidiría el cirenaico con formas aisladas del rodio, délfico y locr. occ., rechazadas por J. Méndez Dosuna, op. cit., p. 173; - a partir de un supuesto ac. pl. *-evऽ, E. Schwyzer, Gr. Gr., I, p. 563; - a partir del nom. pl. de los temas consonánticos y como formas tardias debido a que el nom. ac. pl. de los temas en -èu-coinciden tal y como ocurre en la koiné contemporánea

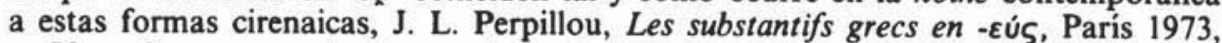
p. 72, n. 3; - por analogía con el ac. pl. de los temas en -t- "noגıs, asi *iapevs, C. J. Ruijgh, Mnemosyne 27, 1-2, 1984, p. 60, n. 10.

${ }_{19}$ Teniendo en cuenta que la wau desapareció en este dialecto en época tempra-

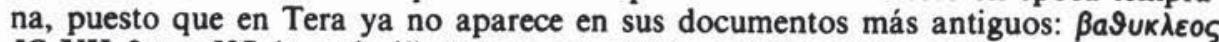
IG XII 3 n. 537 (s. viII), KAeayopas n. 762 (s. viI) (los datos de Cirene anteriores al s. IV son escasos y de dificil interpretación, cf. C. Dobias-Lalou, $R P h$ 96, 1970, pp.

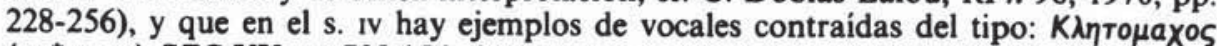

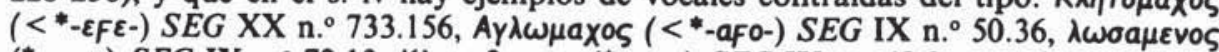

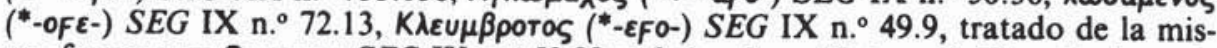

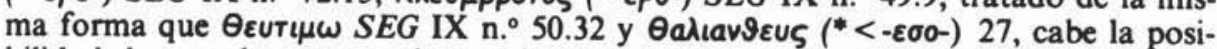
bilidad de postular que en el $\mathrm{s}$. Iv el nom. ac. de los temas en *-ēu-coincidiría con

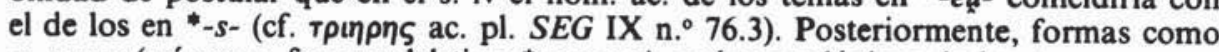
o tapes (así como formas del tipo *tpınpes), serían analógicas de los temas consonánticos. El ac. pl. ıарєs coincidiría a partir de este momento con el nom. pl., de la

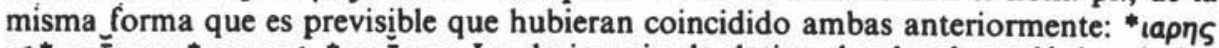

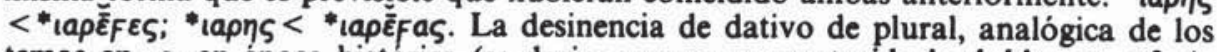
temas en $-s$ - en epoca historica (es decir, una vez reconstruida la doble $-s s-$, cf. A.

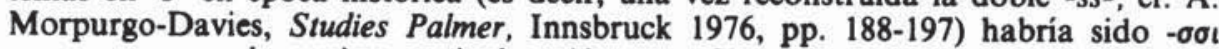

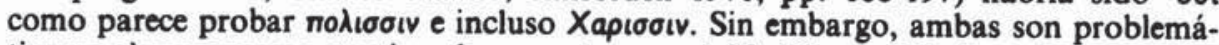

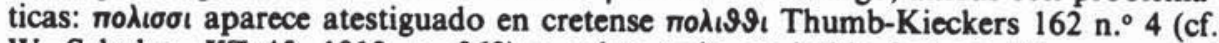

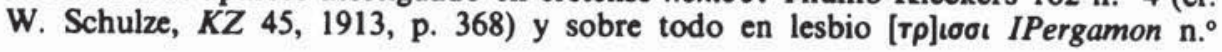




\section{La forma de infinitivo $\delta\llcorner\delta \omega v$}

En la inscripción $S E G$ IX n.$^{\circ} 4(16 / 5)$ de Cirene se atestigua el infinitivo $\delta \iota \delta \omega v 40$, equiparable con exactitud a la forma lesbia ${ }^{20} \delta \iota \delta \omega v I G$ XII $2 n .^{\circ} 489.15$. En la misma inscripción cirenaica aparece también

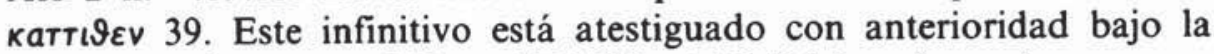
forma «regular» партเ $\vartheta[\varepsilon] \mu \varepsilon v ~ S E G$ IX n. ${ }^{\circ} 72.118$ (s. Iv), por lo que кar$\tau(\vartheta \varepsilon v$ debe entenderse como una forma tardía y secundaria en la que la

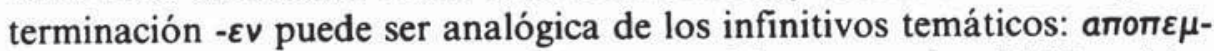

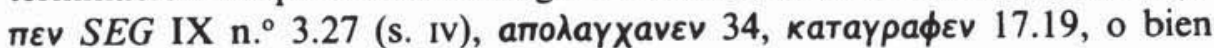
de la de los infinitivos de los verbos contractos ${ }^{21}: \pi \lambda \varepsilon v S E G$ IX n..$^{\circ} 3.28$,

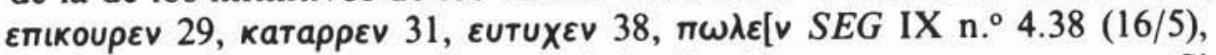

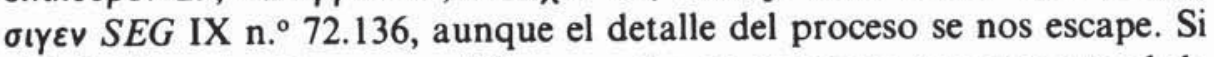
el infinitivo de $T\{\vartheta \eta \mu \iota$ era $T(\vartheta \varepsilon \mu \varepsilon v$ en el s. IV, hay que suponer que el de $\delta i \delta \omega \mu \iota$ era $\delta \iota \delta \delta \mu \varepsilon v$, en cuyo caso $\delta \iota \delta \omega v$ sería, de la misma forma que катт $\vartheta \varepsilon v$, tardío y sólo puede explicarse a partir del infinitivo de los ver-

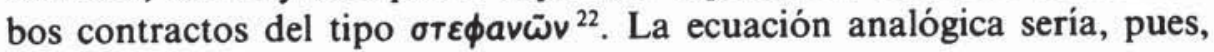

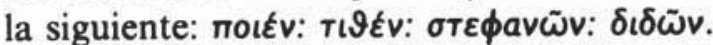

Las flexiones de los verbos contractos han confluido en cirenaico en algunas de sus formas ya desde el s. IV tanto por factores morfo-analógicos como puramente fonéticos: (a) el vocalismo breve - $\varepsilon v$ de los infinitivos temáticos se ha extendido también a las formas medias en los ver-

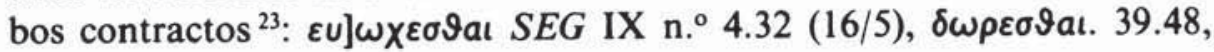

245.80, en donde no puede explicarse a partir de los temas en $-s-$. Por otra parte,

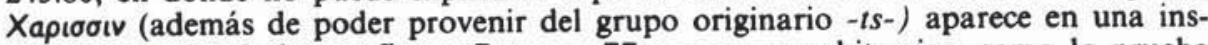
cripción en donde las grafias $\langle\Sigma\rangle$ y $\langle\Sigma \Sigma\rangle$ parecen arbitrarias, como lo prueba y op. cit., p. 452.

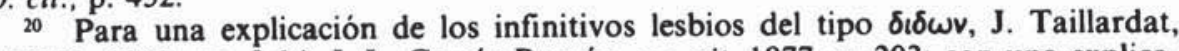
REG 73, 1960, pp. 8-14; J. L. García Ramón, op. cit. 1977, p. 203; con una explicación diferente, W. Blümel, op. cit., p. 209.

${ }_{21}$ Estas formas de infinitivos de verbos contractos también aparecen en el dialecto de Tera (Thumb-Kieckers 180, 14.b), el de Cos (ibid. 203, 27.b), el de Calimna (ibid.), el arg. occ. (ibid. 122, 26.a) y en cretense or., occ. y central (cf. M. Bile, op. cit., p. 165). Para una explicación de estos infinitivos, J. L. García Ramón 1977, op. cit., pp. 201-202.

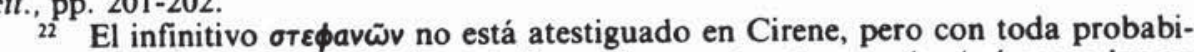
lidad pudo existir. Los infinitivos de los verbos contractos en - $6 \omega$ (así como los en $-\hat{\omega} \omega)$ no conocieron formaciones en *-ov o *av, J. L. García Ramón 1977, op. cit., p. 202 , n. 75. G. Devoto, sin embargo, ha querido ver un infinitivo en -ov en la for-

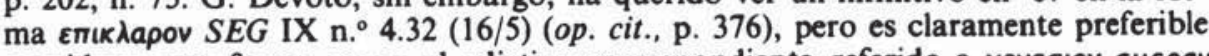

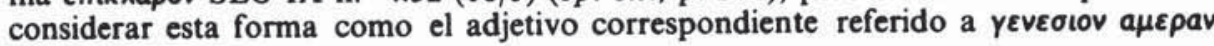
32.

23 Asi lo explica J. L. García Ramón 1977, op. cit., p. 203: «Le dialecte cyrénéen pousse jusqu'à ses dernières conséquences la tendence analogique à la formation d'infinitifs brefs dans les verbes 'contractes', déjà sans le moindre rapport avec 


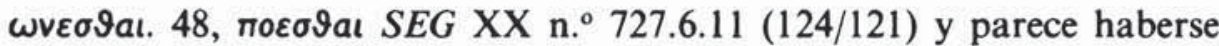

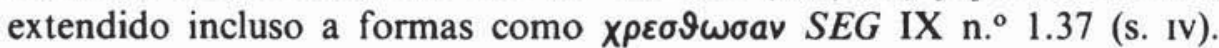
(b) Al menos desde el s. III aparecen formas de participios de presente en las que el grupo -eont- evoluciona fonéticamente a -ent-24: $\delta a \mu \varepsilon \rho-$

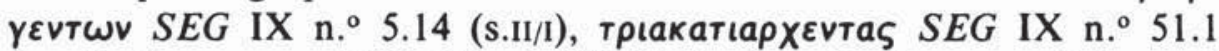
(s. III), ง

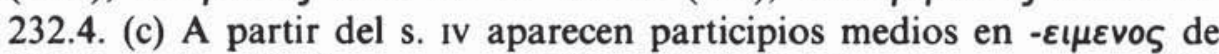

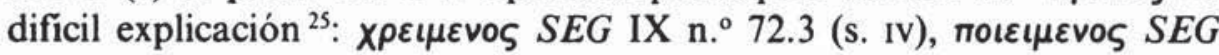

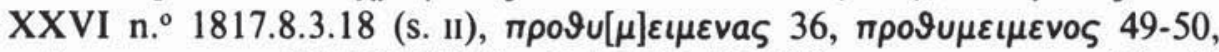

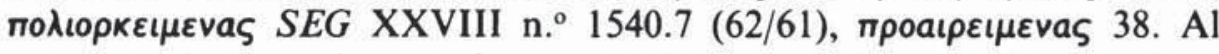
margen del origen de estas formas en cirenaico y de la realidad fonética que recubran las grafias, lo cierto es que aparentemente se desvian de las formas temáticas esperables en $-\varepsilon \circ \mu \varepsilon v o \varsigma\left({ }^{*}-\varepsilon \cup \mu \varepsilon v o \varsigma\right)$.

Todo ello indica que la tendencia a la regularización del paradigma (a) y quizá (c) y determinados procesos fonéticos (b) en los verbos contractos, ha hecho que, al menos desde el s. IV, ambas flexiones verbales coincidan formalmente en determinados casos. Este paralelismo pudo influir en el hecho de que fueran precisamente los infinitivos contrac$\operatorname{tos}^{26}$ los que influyan analógicamente en los de los atemáticos: algo se-

l'action de la loi d'Osthoff en sandhi». Para una opinion diferente, cf. C. J. Ruijgh 1984, op. cit., p. 72, n. 29.

${ }_{24}$ Estas formas no deben ser entendidas como un resto de la flexión atemática de estos verbos, sino que responden a una evolución fonética, como lo demuestra

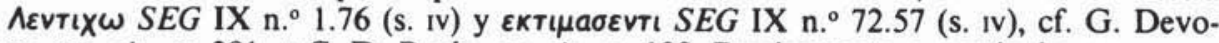
to, op. cit., p. 391, y C. D. Buck, op. cit., p. 132. Precisamente a partir de $\varepsilon \kappa r t \mu a \sigma \varepsilon v-$ Tı podría explicarse la extraña forma de futuro nотоıєৎ $S E G$ XX n. 719.10 (s. II),

${ }_{25}$ Para los participios en - $\varepsilon t \mu \varepsilon v o s, \mathrm{cf}$. H. Hock, The so-called Aeolic inflection of the Greek contract verbs, Ph. D., Yale Univ. 1970, pp. 359-360, y en general, para todo lo relacionado con los verbos contractos. Ahora bien, la presencia de estos participios fuera del ámbito de los dialectos nordoccidentales debe entenderse como influencia de éstos, cf. J. Méndez Dosuna, op. cit., p. 495. La situación en cirenaico es compleja: en dos de las tres inscripciones en las que aparecen hay una fluctuación

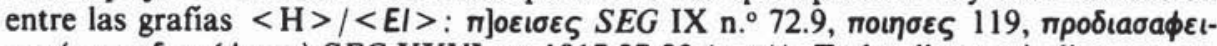
$\sigma \iota v(=\pi \rho \circ \delta i a \sigma a ́ \phi \eta \sigma \iota)$ SEG XXVI n. ${ }^{\circ} 1817.27-28$ (s. $\left.11 / 1\right)$. Todo ello nos indica que estos ejemplos pueden recubrir grafias no fonéticas.

${ }_{26}$ También los infinitivos cretenses de la ciudad de Gortina en - $\mu \eta \nu$ han sido explicados a través de los de los verbos contractos, así M. Bile, BSL 70, 1975, p. 174 (cf. sin embargo, J. L. García Ramón 1977, op. cit., p. 187, n. 33). Con todo, las interferencias entre las formaciones de los infinitivos son frecuentes y, en general, la flexión temática tiende a invadir los dominios de la atemática, cf. J. L. Garcia Ramón, ibid., p. 186, n. 27. Por lo que respecta al infinitivo cirenaico $\delta \iota \delta \omega v$ también C. D. Buck, The Greek Dialects, Chicago 1973, p. 125, lo incluye en el epigrafe "Transfer of $-\mu t$-Verbs to the Type of Contract Verbs" junto con karti $\vartheta \varepsilon v$, sin ningún tipo de explicación. En todo caso, lo realmente llamativo en el caso del cirenaico habria sido la aparición de una forma del tipo * $T$ $\vartheta \eta v$ que no habríamos podido explicar a través de los verbos contractos, ya que éstos, al menos desde el s. Iv, tenían - $\varepsilon v$ como formación de infinitivo. 
mejante a lo que postulamos ha debido de ocurrir en jonio en donde

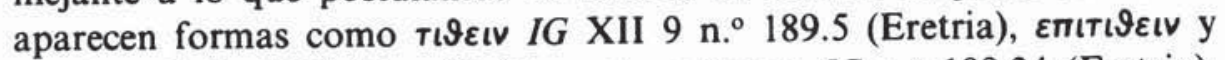

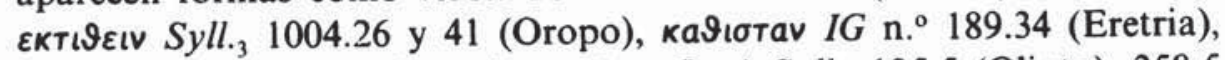

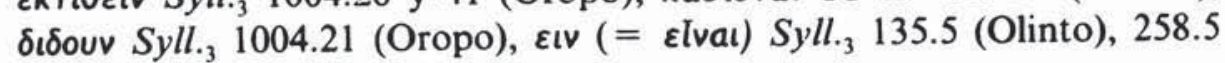
(Oropo), IG 220.8 (Eretria) ${ }^{27}$.

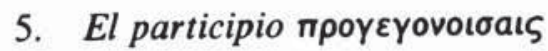

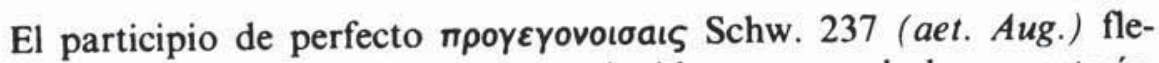
xionado como un tema de presente coincide con una de las características exclusivas ${ }^{28}$ del grupo eolio y más concretamente con el lesbio ${ }^{29}$

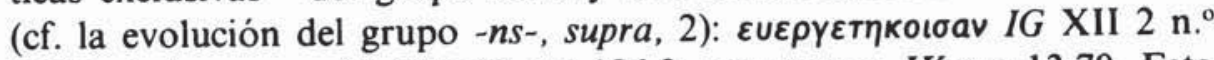

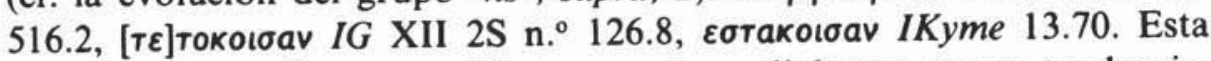
característica eolia se manifiesta en otros dialectos como tendencia, puesto que no aparece nunca de forma sistemática; estos dialectos atestiguan formas de perfecto (pero no participios) conjugadas como temas

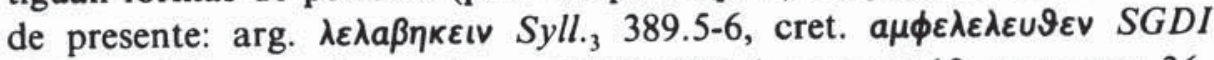

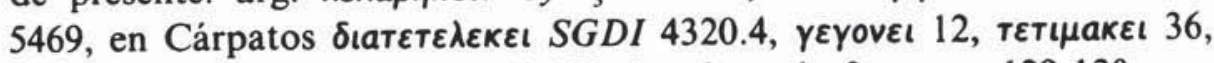

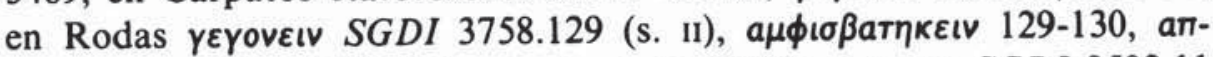

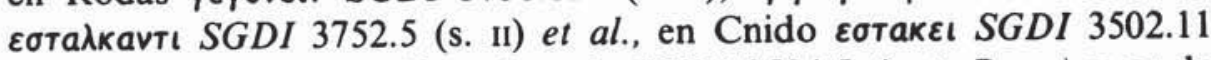

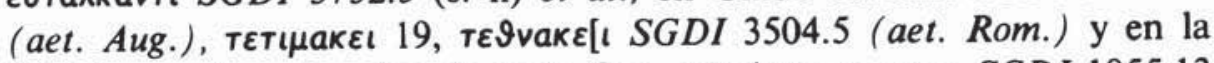

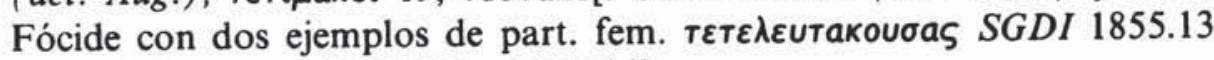

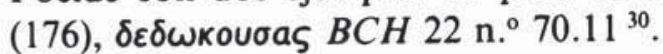

El cirenaico no se aparta de estos últimos dialectos, puesto que en época anterior aparecen participios de perfecto con su propio sufijo:

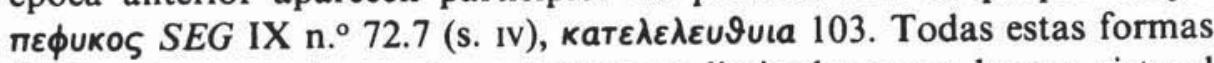
flexionadas como temas de presente pero limitadas como hemos visto al part. fem. y otras formas (no al part. masc.) reflejan una tendencia a la adecuación del tema de perfecto al de presente, pero son esporádicas y no regulares como en los dialectos eolios en los que tanto el part. fem.

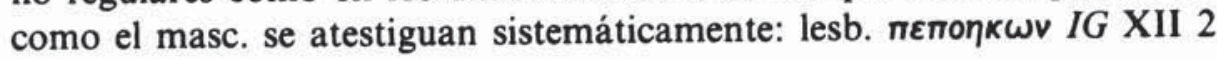

\footnotetext{
27 Thumb-Scherer 280, 25.

28 Los participios de perfecto flexionados como presente tanto femeninos como masculinos se documentan con abundancia en los dialectos eolios, pero la caracteristica exclusiva de este grupo dialectal es el participio masculino de perfecto flexionado como tema de presente, cf. P. Wathelet, op. cit., p. 326; J. L. García Ramón, CFC 5, 1975, p. 269.

${ }_{99}$ W. Blümel, op. cit., p. 228.

30 Para el caso del argólico, Thumb-Kieckers $121 \mathrm{n}$. 22; el cretense, ibid. $166 \mathrm{n}$ 39a; Cárpatos, ibid. 192 n. 20; Rodas ibid.; Cnido, ibid. 202 n. 23; Delfos, 275 n. 31.
} 


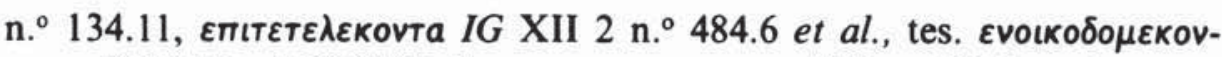

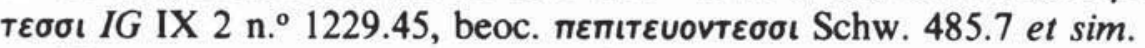

Hay que tener en cuenta, además, que el cirenaico generalizó -oıra/ -aıa como sufijo de participio femenino sustituyendo formas más anti-

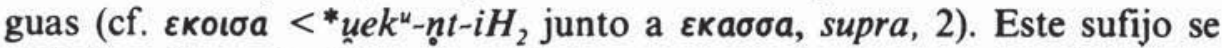
utilizó en época tardia en inscripciones que pretendían resaltar de forma artificial el carácter cirenaico de las mismas ${ }^{31}$, mediante la repetición de alguna de sus características más llamativas como -oıra/aıra o el nom.

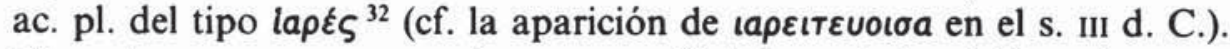
El part. проүєүovoıraıs sería, pues, artificial, es decir, deliberadamente dialectalizante.

\section{Conclusiones}

Hemos pretendido a lo largo de este artículo negar la existencia de un elemento lesbio en Cirene o al menos plantear dudas en cada uno de los rasgos aducidos al respecto:

(a) La evolución del grupo $-n s$ - secundario no ha de ser explicada necesariamente como debida a la presencia de un elemento lesbio en cirenaico que difiere con respecto a aquel dialecto en su tratamiento.

(b) Las formas en - $\varepsilon \sigma \sigma \iota$ de los temas en -èu- no concuerdan con las del lesbio ni se dan de forma regular en otros temas como ocurre en este dialecto.

(c) El infinitivo $\delta\llcorner\delta \omega v$ es una forma tardía y analógica de los ver-

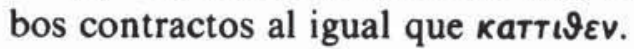

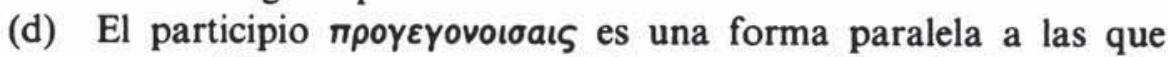
aparecen esporádicamente en otros dialectos no eolios e incluso puede tratarse con mucha probabilidad de una forma artificial.

El precario número de ejemplos que se aducen para justificar la presencia de un elemento lesbio en Cirene para cada una de las características en las que ambos dialectos coinciden, así como los problemas que todos ellos conllevan, inducen, cuando menos, a cuestionar la verosimilitud de la hipótesis, al menos hasta la aparición de nuevos datos sobre este dialecto.

\section{Araceli Striano Corrochano}

${ }^{31}$ Asi, M. Lejeune, BSL 34, 1933, p. 168.

32 Como en $S E G$ XXVI n. ${ }^{\circ} 1817.66$ y $S E G$ XXVIII $1540.13(62 / 61)$. 